\title{
El mercado de la vivienda. Características y principales líneas de actuación pública
}

\author{
Alfonso Fernández Carbajal *
}

\section{El mercado de la vivienda}

\subsection{Características singulares del bien vivienda}

A la hora de analizar el mercado de la vivienda, resulta conveniente comenzar especificando una serie de características que este bien posee y que lo distinguen de la mayoría de los bienes y servicios intercambiados en la economía, ya que el conocimiento previo de las mismas facilita la posterior identificación de las distintas variables que determinan tanto su oferta como su demanda. De entre todas ellas, pueden destacarse las siguientes ${ }^{1}$ :

- Su necesidad, dado que constituye un bien de consumo esencial que ofrece a sus usuarios una serie de servicios imprescindibles para la realización de buena parte de sus actividades cotidianas y, por tanto, para el desarrollo de una vida satisfactoria. Esta circunstancia hace que sea considerado como un bien preferente (ALBI, GonZÁleZ-PÁRAMO y ZuBIRI, 2000, p. 317).

- Su durabilidad, muy superior a la de la mayor parte de las mercancías. Esto implica que es capaz de ofrecer una corriente de servicios de forma gradual durante un largo período de tiempo (FALLIS, 1985, pp. 6-9).

- Su largo periodo de producción, que suele situarse generalmente en torno a dos años como promedio. A este respecto, hay que tener en cuenta que en dicho período debe incluirse no sólo el tiempo empleado en la construcción del inmueble, sino también el necesario para la elaboración de los planos, obtención de los permisos correspondientes, búsqueda de la financiación adecuada y otros similares. Además, la proporción que supone el flujo anual de viviendas terminadas en relación al stock existente es, generalmente, bastante reducida (JAÉN y Molina, 1995, p. 10).

- Su alto coste de construcción, debido en parte a la característica anterior y también al elevado volumen de inputs que requiere su producción. De ello deriva el elevado precio que, en todo caso, adquiere en el mercado en sus diversas formas de tenencia (propiedad, alquiler, etc.), lo que le hace ocupar un lugar preeminente en el presupuesto de gastos de toda unidad familiar. Por tanto, las decisiones que a este respecto se adopten tendrán siempre especial relevancia.

- Su beterogeneidad multidimensional, ya que las viviendas suelen diferir en importantes características cuantitativas y cualitativas. Entre ellas cabe citar, de un modo especial, la localización, dado su carácter espacialmente fijo — salvo excepciones, no pueden ser trasladadas de un lugar a otro-, y la imposibilidad física de que dos viviendas ocupen simultáneamente el mismo espacio. Por tanto, desde el punto de vista económico, una unidad de vivienda incluye una variada «cesta de características» cuya valoración por parte de oferentes y demandantes tenderá a reflejarse, en última instancia, en el precio de la misma $^{2}$.

Estas características «especiales» de la vivienda determinan, en buena medida, el funcionamiento de su mercado o, más 
bien, de sus mercados, dado que puede ser segmentado utilizando distintos criterios - forma de tenencia, edad, localización, grado de intervención pública en su construcción y financiación, etc.-, de los que resulta que se trata más bien de un conjunto de mercados íntimamente relacionados (JAÉN y Molina, 1995, p. 9). A este respecto, hay que destacar que la ya citada fijación espacial de la vivienda hace que su mercado se vea muy influido por el contexto social, económico y político del área geográfica en que se encuentran enclavadas, lo que obliga a considerarlo como un conjunto de mercados locales más o menos interrelacionados y a que, en muchas ocasiones, haya que circunscribir el análisis a áreas urbanas concretas. Ello sin perjuicio de que haya que ampliar dicho ámbito geográfico para analizar los efectos de determinados factores que inciden sobre varios de ellos conjuntamente, lo que permitirá apreciar tendencias generales en la evolución de los mismos - a nivel de una región o país, por ejemplo-.

De entre todos los criterios citados, también merece destacarse por su especial trascendencia el referido a la forma de tenencia, el cual nos permite distinguir básicamente entre el mercado de vivienda en propiedad y el mercado de vivienda en alquiler. Ambas modalidades permiten satisfacer las necesidades de vivienda que puedan tener las familias, por lo que desde este punto de vista son sustitutivas. Por otra parte, a la hora de elegir entre una $u$ otra hay que tener en cuenta que ambas presentan ventajas e inconvenientes contrapuestos. Así, la principal ventaja de la propiedad es la seguridad que ofrece a su titular ante la posibilidad de que sus ingresos futuros no le permitan hacer frente a los desembolsos necesarios para el pago de los alquileres, bien sea por un incremento inesperado de éstos o por una disminución de aquéllos (ALBI, GoNZÁLEZ-PÁRAMO y ZUBIRI, 2000, p. 257). Por contra, las principales ventajas del alquiler frente a la propiedad radican en el menor desembolso inicial que es necesario realizar para acceder a la misma y los menores costes de transacción que suele implicar el cambio de vivienda (Mills y Hamilton, 1994, pp. 233-234). Partiendo de estas consideraciones iniciales, las familias elegirán la modalidad de tenencia teniendo en cuenta varios factores. Concretamente, tenderá a ser más ventajosa la compra frente al alquiler cuanto mayor sea el número de años que dicha familia espera permanecer en la vivienda, cuanto mejores condiciones ofrezca el crédito hipotecario que necesite y cuanto mejor sea su tratamiento fiscal comparado con el de la otra forma de tenencia (Mills y Hamilton, 1994, pp. 234-236).

\subsection{La demanda de vivienda}

En lo que a la demanda se refiere, y centrándonos sobre todo en el mercado de vivienda en propiedad, sus principales factores determinantes son, aparte del propio precio de esta mercancía, los siguientes:
- La evolución de la población y, más concretamente, del ritmo de creación de nuevos hogares, que, a su vez, depende de variables tales como el crecimiento vegetativo de la población o los posibles movimientos migratorios existentes. Todo ello está íntimamente relacionado con el ya citado carácter necesario que tiene este bien.

- La evolución de la renta real disponible de las familias. Esto es debido a que su alto coste suele hacer necesario diferir los pagos derivados de su adquisición durante un largo período de tiempo. De este hecho se derivan dos importantes consecuencias:

a) Las decisiones referidas a la demanda de vivienda efectuadas por los hogares no suelen verse afectadas por cambios temporales en su nivel de renta, sino por cambios permanentes, por lo que una definición de renta que tenga en cuenta esta circunstancia (que habitualmente suele aproximarse a través del cálculo de la renta media durante un determinado período de tiempo) es la más adecuada para estudiar la relación entre ambas variables. En este sentido, las estimaciones realizadas sobre la elasticidad-renta de la demanda de vivienda parecen indicar, como por otra parte cabía esperar, que existe una relación positiva entre ellas, aunque con un valor para dicha elasticidad ligeramente inferior a la unidad (MLLS y HamiltoN, 1994, p. 209; Fallis, 1985, p. 33). Esto implica que al aumentar la renta también lo hace, pero en una proporción menor, la cantidad demandada de vivienda; por lo tanto, el porcentaje del presupuesto familiar que supone este gasto tenderá a decrecer, algo que parece confirmarse al calcularlo para familias de renta alta y baja y comparar los resultados obtenidos. Precisamente, el valor de dicho porcentaje suele utilizarse para determinar cuándo una familia tiene un problema de accesibilidad a la vivienda, es decir, dificultades para satisfacer sus necesidades a este respecto. Habitualmente suele considerarse que existe dicho problema cuando los gastos en vivienda de una familia superan el $30 \%$ de su renta disponible (MCDonaLD, 1997, p. 205).

b) Por otra parte, en el ya citado contexto de largo plazo en el que se insertan las decisiones referentes a la vivienda juegan un papel muy importante las expectativas, sobre todo las referidas a la evolución de la coyuntura económica general del país en cuestión, dada la influencia que ésta tiene sobre la renta esperada de las familias. Concretamente, si las citadas expectativas son desfavorables o existe algún tipo de incertidumbre al respecto, la demanda de vivienda suele disminuir significativamente, al producirse un aplazamiento de las decisiones relativas a esta materia. Por el contrario, cuando las expectativas se tornan favorables, la adopción de tales decisiones aplazadas tenderá a provocar un incremento significativo de la misma.

No debemos olvidar tampoco la influencia que la variable renta ejerce en el proceso de creación de nuevos hogares. En 
este sentido, hay que tener en cuenta que la decisión de crear una familia y adquirir una vivienda suelen adoptarse frecuentemente de forma conjunta. Por tanto, podemos decir que la citada variable influye en la demanda de vivienda tanto de forma directa - a través de las decisiones de las familias ya constituidas - como indirecta - a través de las decisiones relativas a la creación de nuevos hogares-.

- La evolución de los mercados financieros y las medidas de política monetaria. El ya aludido alto coste de la vivienda provoca que, para su adquisición, las familias se vean generalmente obligadas a acudir a los mercados financieros a fin de pedir prestados los fondos necesarios para hacer frente a una parte importante de su precio. Es evidente, por tanto, que las características de los créditos hipotecarios (cuantía permitida, tipos de interés, plazo de amortización) son elementos fundamentales a la hora de decidir la adquisición de una vivienda. Éstas, a su vez, suelen depender de dos variables íntimamente ligadas entre sí: la situación general de los mercados financieros, dada la interrelación existente entre todos ellos, y las medidas de política monetaria adoptadas por los bancos centrales, que tradicionalmente han tenido una importancia destacada en lo que a la evolución de los tipos de interés y el volumen de crédito disponible se refiere, variables ambas que resultan decisivas para explicar las variaciones experimentadas por la demanda de vivienda según algunas estimaciones (RodRíguez, 1982, p. 188). Por otra parte, la evolución de los mercados financieros es una variable importante por otra razón fundamental: la vivienda, como es bien sabido, se comporta también como un activo (como un bien de inversión y no sólo de uso), y como tal compite con los propiamente financieros por el ahorro generado en la economía de un país. Parece, por tanto, evidente que cuanto mayores sean las expectativas de rentabilidad en el mercado inmobiliario en comparación con las que ofrezcan los mercados financieros mayor peso tendrá este componente de la demanda, lo que además tenderá a sesgar en favor de la propiedad la modalidad de tenencia elegida por las familias. Si las citadas expectativas fuesen menores ocurriría, lógicamente, lo contrario.

\subsection{La oferta de viviendas}

Refiriéndonos ahora a la oferta, hay que destacar la relación que ésta guarda con el precio de la vivienda. A este respecto, conviene recordar una de las características de esta mercancía señaladas anteriormente: su largo período de producción. Esto hace que, ante una variación en el precio de la vivienda, los efectos sobre la cantidad ofrecida no puedan apreciarse a corto plazo, sino a largo plazo, ya que un aumento o reducción del número de viviendas iniciadas sólo se verá reflejado en el mercado cuando éstas se terminen y se pongan a la venta, necesitándose a veces un flujo importante de viviendas durante varios años para que dichos efectos sean significativos. En defi- nitiva: la oferta de viviendas es inelástica a corto plazo. Además, esta inelasticidad puede manifestarse también a más largo plazo si escasea uno de los inputs fundamentales para su producción: el suelo urbano. En este caso, si se produce un fuerte incremento de la demanda la subida de los precios será más intensa y duradera, ya que la oferta será incapaz de ajustar el mercado vía cantidades no sólo a corto, sino también a largo plazo.

Por otra parte, y como actividad económica que es, la vivienda es uno de los componentes, junto con la edificación no residencial y la obra civil, de la rama de la construcción, que, generalmente, aparece separada en las estadísticas oficiales del resto de las actividades industriales debido a que posee una serie de características peculiares que la diferencian claramente de estas últimas. Entre ellas, merecen destacarse dos (SANTIAGO DE PABLO, 1982, p. 164): su carácter «artesanal», ya que generalmente ha de elaborar las unidades que produce «a medida», $y$, en parte debido a lo anterior, la importante cantidad de trabajo que contrata y que la convierte, a pesar de las innovaciones tecnológicas introducidas, en la actividad industrial que más mano de obra absorbe. A esto hay que añadir que se trata de una actividad inductora de otras muchas, tales como las dedicadas a la producción de materiales de construcción -los llamados «efectos de arrastre bacia atrás»- o de bienes y servicios que constituyen los equipamientos básicos de las viviendas construidas — «efectos de arrastre bacia adelante»- (MARTínEZ, 1982, p. 122$)^{3}$.

\subsection{El funcionamiento del mercado de la vivienda: ciclos e imperfecciones}

En lo referente al funcionamiento del mercado de la vivienda hay que destacar, en primer lugar, que evoluciona de forma cíclica $y$, en segundo lugar, que presenta una serie de imperfecciones que le alejan del modelo competitivo. Vamos a analizar estos dos aspectos con más detalle:

\subsubsection{Los ciclos en el mercado de la vivienda}

El origen de los mismos hay que buscarlo en algunos de los ya citados rasgos que caracterizan tanto a la oferta -inelástica a corto plazo - como a la demanda - bastante sensible a las expectativas relacionadas con la evolución de la coyuntura económica general-. El resultado de todo ello es que cuando la coyuntura económica se torna desfavorable la demanda de vivienda disminuye bruscamente - las familias aplazan la satisfacción de sus necesidades de vivienda, dadas sus menores expectativaś de renta y el elevado precio de esta mercancíaen tanto que la oferta no podrá adaptarse a la misma de forma inmediata, ya que las viviendas terminadas que salen al mercado en ese momento se iniciaron antes del cambio experimentado 
por dicha coyuntura. La consecuencia inmediata, al igual que ocurre en cualquier otro mercado, es la aparición de un importante exceso de oferta de viviendas. En una situación como ésta, las leyes de la oferta y la demanda predicen que el precio de la mercancía debería descender hasta que se alcance un nuevo equilibrio entre ambas. No obstante, lo habitual es que el ajuste vía precios no sea completo: generalmente se suele producir un estancamiento o ligera disminución de los mismos en términos nominales ${ }^{4}$. Como consecuencia de esta relativa rigidez a la baja, el desequilibrio oferta-demanda no se corrige totalmente, apareciendo un stock de viviendas sin vender más o menos importante en función de la gravedad de la recesión económica.

$\mathrm{Si}$, al contrario que en el caso anterior, la coyuntura se torna favorable, se produce la situación inversa: un importante incremento de la demanda - muchas familias se deciden ahora a satisfacer sus necesidades de vivienda aplazadas durante la crisis- en tanto que la oferta de viviendas terminadas es la planificada cuando la coyuntura era más desfavorable. El resultado de todo ello es la aparición de un exceso de demanda en el mercado ante el cual las precios tenderán a incrementarse de forma significativa, dando lugar en ocasiones, cuando este proceso inflacionista es especialmente intenso, a lo que habitualmente se conoce como «boom inmobiliario». Un ejemplo de este fenómeno lo tenemos cuando analizamos la evolución de este mercado en nuestro país durante la segunda mitad de los ochenta (RAFOLS I EsTEVE, 1989, pp. 90-93).

Nos encontramos, por tanto, con unos ciclos en este mercado que evolucionan afectados por la situación económica general del país. Pero además, dados los ya citados efectos de arrastre que posee esta actividad, la influencia es recíproca, de tal forma que los ciclos inmobiliarios tienden a acentuar las expansiones y los períodos de recesión de la economía en su conjunto, como también se ha podido observar en nuestro país (RODRÍGUEZ, 1982, p. 192) ${ }^{5}$.

\subsubsection{Las imperfecciones en el mercado de la vivienda}

Además de la evolución cíclica analizada con anterioridad, en este mercado están presentes una serie de imperfecciones o «fallos», es decir, rasgos característicos que le alejan del modelo de mercado perfectamente competititivo, que es el ideal des. de el punto de vista económico ${ }^{6}$. Entre ellas cabe citar dos fundamentales:

- La presencia de externalidades (Rosen, 1987, pp. 377-378), es decir, de efectos sobre el bienestar que la producción y/o el consumo de vivienda ocasiona a agentes distintos de sus productores o consumidores originales y que no se reflejan totalmente en los precios de mercado, lo que puede ser una fuente de ineficiencias. Ejemplos de externalidades habitualmente mencionados son los efectos positivos que la rehabilitación exterior de una vivienda ejerce sobre las que la circundan, o los negativos que generan los barrios degradados, aunque, en este caso, es discutible si es la vivienda o la baja renta de sus moradores - que les dificulta el hacer frente a los gastos de mantenimiento de la misma - la que, en última instancia, genera tales externalidades. En todo caso, el resultado es que, al no tenerse en cuenta tales efectos, la producción y/o consumo de vivienda no será el «óptimo» desde el punto de vista social: se rehabilitarán menos viviendas y persistirán más viviendas degradadas de lo que sería deseable.

- Información imperfecta (FaluIs, 1985, p. 10). El gran número de características que definen una vivienda y que la pueden diferenciar de las demás, la ya aludida heterogeneidad multidimensional, hace que sea difícil y costoso obtener una información detallada sobre cada una de las viviendas ofertadas en el mercado, por lo que la existencia de información imperfecta suele ser algo habitual en un bien que dista mucho de ser homogéneo. No obstante, la presencia de instituciones especializadas en el negocio inmobiliario (agencias, tasadores, etc.), ha tendido a reducir el grado de imperfección de la información disponible.

Por otra parte, si a los costes de búsqueda añadimos los derivados de la mudanza (molestias ocasionadas, tiempo empleado en realizarla, «capital humano» perdido en forma de amistades, etc.), podemos concluir afirmando que los costes de movilidad no son nada despreciables en este mercado. Esto supone, en la práctica, que sea difícil encontrar una situación de claro equilibrio oferta-demanda en el mismo, de tal modo que algunos autores (Fallis, 1985, pp. 78-79; ARNOTT, 1987, pp. 981-983; SMITH, RoSEn y FaLlis, 1988, p. 50) afirman que, en clara analogía con lo que ocurre en el mercado laboral, existe una «tasa natural de viviendas vacantes» ${ }^{7}$.

\section{Las políticas de vivienda}

\subsection{Los objetivos de la política de vivienda}

Otro de los rasgos que caracterizan el funcionamiento del mercado de la vivienda en la mayoría de los países es el importante grado de intervención pública en el mismo. Esta intervención tiene su razón de ser en el logro de una serie de objetivos que, derivados en última instancia de lo señalado en los apartados anteriores, pueden agruparse del siguiente modo (LEAL, 1990, pp. 16-23). 


\subsubsection{Objetivos macroeconómicos}

Como ya se ha señalado anteriormente, el sector de la construcción juega un importante papel en la economía de un país por dos motivos básicos: su capacidad para generar empleo y sus efectos de arrastre sobre otras actividades productivas. Esto hace que los ciclos que se observan en el mercado inmobiliario tiendan, a su vez, a influir significativamente en los ciclos que caracterizan la evolución de la coyuntura económica general de un país. Por este motivo, la política de vivienda es frecuentemente utilizada como instrumento de política anticíclica en momentos de recesión económica. El objetivo que se persigue es atenuar los ciclos inmobiliarios, es decir, que esta actividad económica mantenga unos niveles de producción aceptables para que, teniendo en cuenta los efectos anteriormente citados, contribuya a atenuar también los de la actividad económica general, en especial en sus fases recesivas o de crisis. En materia de vivienda, los instrumentos de política anticíclica suelen basarse en el establecimiento de subvenciones generalizadas - sobre todo por vía fiscal- $\mathrm{y}$ en la canalización hacia la actividad inmobiliaria de importantes volúmenes de recursos financieros destinados a la concesión de créditos.

Sin embargo, la utilización de la política de vivienda para alcanzar objetivos macroeconómicos no está exenta de críticas. Así, algunos afirman que con esta medida no se consigue reactivar el sector, ya que lo único que se produce es simplemente una sustitución de producción de vivienda no subvencionada por vivienda subvencionada. No obstante, el hecho de que se produzca esta sustitución en momentos de crisis no puede considerarse negativo, ya que ayuda a mantener el nivel de actividad en este mercado, lo que ya es en sí mismo un efecto anticíclico importante teniendo en cuenta lo señalado con anterioridad. De no producirse, lo que puede ocurrir es que la fase recesiva del ciclo sea más intensa.

\subsubsection{Objetivos sociales}

Mediante este tipo de objetivos, lo que se persigue es incrementar el grado de equidad, entendida ésta como el intento de lograr una mayor igualdad en los niveles de bienestar de los individuos que componen la sociedad o al menos ofrecer las posibilidades para que aquélla se produzca ${ }^{8}$. Dada la importancia que, a este respecto, tiene la vivienda (recuérdese que es considerada habitualmente como un bien preferente), lo que se pretende es facilitar el acceso a la vivienda a aquellos individuos con menores ingresos $y$, por tanto, con mayores dificultades para acceder a ella. Para conseguirlo, las medidas que se proponen pasan por subvencionar de alguna forma el consumo de vivienda que realizan estos estratos de población. Como puede observarse, este tipo de ayudas se diferencian de las otorgadas por razones de política macroeconómica en que son selectivas y no generalizadas, es decir, sólo pueden beneficiarse de ellas aquellas familias que no superen un determinado nivel de ingresos. Esta distinción es importante, porque el carácter selectivo o indiscriminado de las ayudas que se otorguen en cada momento resultará esencial a la hora de hacer una valoración de la política de vivienda que se esté desarrollando.

$\mathrm{Al}$ igual que ocurre con los objetivos anteriores, la consecución de un mayor grado de equidad a través de estas medidas está también sometida a un cierto grado de controversia que, pese a todo, no ha impedido que sean habitualmente usadas para este fin. En este sentido, hay que destacar que se trata de transferencias en especie, dado que las ayudas están ligadas al consumo de un bien concreto: la vivienda. Este hecho ha generado un cierto debate entre los economistas por dos motivos fundamentales:

En primer lugar, algunos consideran más adecuadas las transferencias en efectivo, de tal forma que los beneficiarios de las mismas puedan destinar los fondos a aquellos bienes que deseen. De este modo, argumentan, podrían alcanzar mayores niveles de bienestar al satisfacer mejor sus preferencias (AL bi, Gonzalez-Paramo y Zubiri, 2000, pp. 318-319; Rosen, 1987, p. 379). Para otros, sin embargo, es necesario tener en cuenta el importante papel de este tipo de ayudas a la hora de disuadir a los «impostores», es decir, a los individuos que reclaman las ayudas sin necesitarlas realmente (NICHOLS y ZEC. KAUSER, 1982, pp. 372-379). En el caso concreto que nos ocupa, este efecto se logra al obligar a los individuos beneficiarios a destinar las ayudas al consumo de determinadas viviendas que, de hecho, se comportan como bienes inferiores respecto a las demás. De esta forma, los individuos que, por sus niveles de renta, desean acceder a viviendas de estándares más elevados previsiblemente no las solicitarán (JAÉN y MOLINA, 1992, p. 109).

En segundo lugar, también suele ser objeto de críticas este tipo de ayudas por su carácter paternalista (González-Páramo y ONRUBIA, 1992, p. 192), dado que con ellas se trata de alterar los patrones de consumo de los individuos en favor de determinados bienes que el sector público considera más adecuados para ellos, produciéndose, por tanto, una intromisión en su libertad. No obstante, hay que tener en cuenta los ya citados efectos externos que puede generar un insuficiente consumo de vivienda por parte de algunos individuos hacia el resto de la comunidad. Desde este punto de vista, se podría argumentar que se trataría de limitar o influir en las conductas de los individuos que pueden afectar al resto de la comunidad y que, por ello, son susceptibles de limitar los derechos y libertades de ésta. 


\subsubsection{Objetivos urbanísticos}

Como se puede apreciar fácilmente, las viviendas ocupan la mayor parte de la superficie de una ciudad, lo que las convierte en un instrumento clave a la hora de definir la morfología urbana. Por tanto, parece evidente que las políticas de vivienda tienen unas implicaciones urbanísticas importantes. Así, pueden favorecer la regeneración de espacios urbanos degradados, lo que redundará en una mayor eficiencia en la asignación de los recursos en la medida en que corrija las externalidades que se estaban produciendo y en un mayor grado de equidad en la medida en que se favorezca la mejora de las áreas urbanas ocupadas por los individuos con menores niveles de renta ${ }^{9}$.

Por otra parte, hay que tener en cuenta que la política urbanística forma parte de la política de vivienda, entendida ésta en sentido amplio. A este respecto, hay que destacar que la planificación urbana, en la medida en que determina dónde, qué y con qué intensidad se puede edificar, influye claramente sobre la oferta inmobiliaria, de tal forma que cuantas más restricciones se impongan al respecto menos capacidad tendrá aquélla para satisfacer las necesidades de la demanda y, sobre todo, para reaccionar adecuadamente ante un incremento de esta última ${ }^{10}$. Esto no implica, lógicamente, que no deba existir ningún tipo de restricción, dado que un desarrollo urbanístico adecuado hace más habitables las ciudades y, por tanto, permite que sus ciudadanos alcancen mayores niveles de bienestar. Será necesario, pues, el establecimiento de unas normas urbanísticas que compatibilicen, en la medida de lo posible, una aceptable elasticidad de la oferta inmobiliaria con una calidad de vida satisfactoria para los habitantes de las ciudades, algo que no resulta fácil, por otra parte.

\subsection{La instrumentación de las políticas de vivienda}

Todos estos objetivos son los que, habitualmente, se utilizan para justificar la intervención pública en el mercado de la vivienda. Dependiendo de cuál o cuáles sean los considerados como prioritarios, las políticas desarrolladas serán, lógicamente, diferentes. No obstante, en todas ellas se pueden distinguir dos tipos básicos de actuaciones: las fundamentalmente reguladoras y las de carácter eminentemente presupuestario.

\subsubsection{Actuaciones reguladoras}

En este grupo, se incluyen toda una serie de normas establecidas por la Administración para conseguir alguno de los objetivos anteriormente señalados y que afectan bien directamente al mercado de la vivienda o a otros íntimamente relacionados con él. Entre ellas cabe citar las siguientes: a) Actuaciones de ordenación urbana y control de la edificación, mediante las cuales se intenta regular el proceso de producción de nuevas viviendas y, en su caso, la rehabilitación de las antiguas. Entre ellas cabe citar: i) aquellas que tratan de regular tanto la oferta como el uso de uno de los inputs esenciales en esta materia, como lo es el suelo, y ii) las que tratan de determinar los estándares mínimos que debe de reunir un inmueble (calidad de los materiales empleados, instalaciones mínimas de las que debe disponer y otras medidas similares).

b) Medidas de control de alquileres, que pueden adoptar básicamente dos formas: i) congelación total de los mismos, o ii) limitación de los incrementos permitidos según la evolución de algún índice tomado como referencia, generalmente el índice de precios al consumo. Pese a la aparente «bondad» del objetivo perseguido - proteger al inquilino frente a los aumentos de precios que puedan tratar de imponer los propietarios- los resultados suelen ser contraproducentes, sobre todo si la forma de control utilizada es del primer tipo. Esto es debido a que desincentiva el destino de las viviendas a esta modalidad de tenencia, con lo que, al reducirse su oferta, muchas familias no podrán hacer uso de la misma ${ }^{11}$.

c) Medidas de regulación financiera, frente a las cuales el mercado inmobiliario ha manifestado tradicionalmente una especial sensibilidad dada la necesidad de financiación ajena que habitualmente tienen tanto oferentes como demandantes. En este sentido hay que destacar: i) las medidas de control monetario que, aunque afectan al conjunto de la economía del país, tienen unos efectos particularmente importantes en este mercado al constituir un referente fundamental respecto a la evolución de los tipos de interés y del volumen de crédito disponible, y ii) las específicamente vinculadas al mercado hipotecario, de cara a facilitar la canalización de los recursos financieros cuantitativa y cualitativamente necesarios y entre las que cabe citar, a modo de ejemplo, las referidas a las características de los activos financieros emitidos para captar fondos con destino a este mercado -los llamados títulos hipotecarios-o las aparecidas en fechas más recientes referidas a la refinanciación de los créditos hipotecarios mediante la titulización de los mismos (RodrígueZ, 1990, pp. 189-191).

\subsubsection{Actuaciones presupuestarias}

Se incluyen aquí todas aquellas medidas que tienen su correspondiente reflejo en los presupuestos de las administraciones públicas, es decir, las diferentes ayudas económicas — también llamadas subsidios - que, implícita o explícitamente, se conceden para facilitar a las familias el acceso a la vivienda. Estas últimas, a su vez, pueden ser objeto de distintas clasificaciones, según el criterio que se emplee para ello. Entre ellas cabe citar las recogidas a continuación. 
- Según se tengan o no en cuenta las características socio-económicas del adquirente o usuario, a la hora de conceder el derecho a disfrutar de las ayudas, éstas pueden clasificarse en (LEVENFELD, 1989, p. 7):

a) Ayudas «a la piedra», si no se tienen en cuenta y es la vivienda por sí misma la que da derecho a obtener la ayuda siempre que ésta cumpla los requisitos que se exijan para ello.

b) Ayudas «a la persona», aquellas cuya percepción está ligada a las características socio-económicas del adquirente o usuario de la misma, aunque en muchas ocasiones también se exige que la vivienda en cuestión reúna determinadas características.

Como se puede observar, las ayudas a la piedra tienen un carácter más indiscriminado que las ayudas a la persona, por lo que suelen ser utilizadas generalmente con fines macroeconómicos. No obstante, también se las puede dotar de un cierto carácter social. Para ello, basta limitar las ayudas a aquellas viviendas que, por sus características (precio por metro cuadrado y superficie relativamente reducidos, por ejemplo) se comporten de hecho como bienes inferiores respecto al resto de las viviendas construidas. De esta manera, se desincentivaría la adquisición de las mismas por los individuos con mayores niveles de renta.

- Por otra parte, según sea el reflejo que las ayudas tengan en el presupuesto público, éstas se pueden clasificar en (GoN. ZÁlEZ-PÁrAMO y ONRUBIA, 1990, pp. 198-202):

a) Ayudas directas, que son aquellas que se conceden vía presupuesto de gastos, es decir, suponen un gasto directo para la Administración pública. Generalmente, suelen adoptar dos formas: reducciones en el precio de la vivienda ${ }^{12}$, mediante la concesión de una subvención de cuantía equivalente a un porcentaje del precio de la misma, o en el tipo de interés del préstamo solicitado, lo que habitualmente se conoce como subsidiación de intereses.

b) Ayudas indirectas, aquellas que se conceden vía presupuesto de ingresos y que suponen una minoración de los mismos. Se trata, fundamentalmente, de ayudas de carácter fiscal, es decir, que suponen para el beneficiario la rebaja o exención en el pago de determinados impuestos. Entre las medidas de este tipo habitualmente utilizadas en la mayor parte de los países desarrollados hay que destacar la posibilidad de deducir en el impuesto sobre la renta personal los intereses de los créditos utilizados en la adquisición del inmueble.

En general, las ayudas indirectas o fiscales son utilizadas por las Administraciones públicas para configurar una política de vivienda dirigida a incentivar la demanda de los estratos de población con ingresos medios y altos. Su mantenimiento suele justificarse frecuentemente argumentando que, de esta forma, se logra que el sector de la construcción -importante en la economía de un país por las razones señaladas con ante- rioridad - mantenga unos aceptables niveles de actividad. Por el contrario, las ayudas directas suelen ser el instrumento utilizado para facilitar el acceso a la vivienda a las familias de bajos ingresos, lo que habitualmente suele denominarse como politica social de vivienda ${ }^{13}$. Por tanto, podemos decir que, en relación a las dos clasificaciones vistas hasta ahora, parece existir una tendencia clara a que las ayudas indirectas sean «a la piedra» mientras que las ayudas directas suelen ser las que habitualmente se personalizan.

- Por último, teniendo en cuenta el lado del mercado a través del cual se canalicen las ayudas, éstas se pueden clasificar en (McDonald, 1997, pp. 226-228; Mills y Hamilton, 1994, pp. 266-267) ${ }^{14}$ :

a) Ayudas por el lado de la oferta, que son aquellas destinadas a favorecer la construcción de viviendas bien por organismos públicos o por agentes privados. En este apartado, habría que incluir la promoción pública de viviendas, mediante la cual el sector público se involucra en la construcción de viviendas destinadas a las familias con menores ingresos, y las ayudas que se conceden a los promotores privados, condicionadas en ocasiones a que el destino y/o destinatario de las mismas sea el establecido por la Administración —alquiler, individuos de renta baja u otros similares-.

b) Ayudas por el lado de la demanda, aquellas concedidas directamente a las familias para facilitar su acceso a la vivienda.

En relación con esta última clasificación, conviene destacar dos aspectos importantes. En primer lugar, la no equivalencia exacta entre ayudas «a la piedra» y subsidios por el lado de la oferta, por una parte, y ayudas «a la persona» y subsidios por el lado de la demanda, por otra. Así, por ejemplo, en la medida en que las ayudas a la oferta estén condicionadas a que las viviendas construidas se destinen a familias de bajos ingresos y a precios inferiores a los de mercado se están personalizando, de hecho, las ayudas. En segundo lugar, la promoción pública de viviendas es cuestionada por algunos autores, entre otras razones, al considerar que su producción es más cara - $y$, por tanto, más ineficiente- que la privada y por el escaso número de beneficiarios en relación con las necesidades, al no ser generalmente suficiente la cantidad producida de las mismas ${ }^{15}$. En general, estas y otras consideraciones parecen hacer más deseables los subsidios por el lado de la demanda (López, 1991, p. 45; Mills y Hamilton, 1994, p. 269).

Finalmente, un aspecto que es preciso tener muy en cuenta a la hora de establecer un sistema de ayudas -0 de analizar los efectos de uno ya vigente- es la posibilidad de que éstas beneficien no tanto a las familias directa o indirectamente destinatarias de las mismas como a otros agentes que intervienen en el negocio inmobiliario. Esto sucederá si dichas ayudas se traducen finalmente en un incremento de los precios de las viviendas (tanto de venta como de alquiler) de tal forma que 
buena parte del beneficio se traslade a los oferentes de tales viviendas (promotores-constructores, familias con patrimonio inmobiliario y otros). La posibilidad de que se produzca esta traslación por «capitalización» del incentivo, como también se conoce a este fenómeno, dependerá, en buena medida, de tres factores esenciales:

a) El grado de elasticidad de la curva de oferta de este mercado (WHITE y WHITE, 1977, pp. 111-114). A este respecto, hay que señalar que el sistema de subsidios a la vivienda provoca un incremento de la demanda de este bien, puesto que facilita a las familias el acceso a este mercado ${ }^{16}$. Teniendo esto en cuenta, cuanto más inelástica sea la curva de oferta mayor será la subida que se produzca en los precios y, por tanto, mayor será el grado de capitalización que se produzca.

b) La existencia de limitaciones en cuanto al precio - de venta o alquiler- de las viviendas a las que se pueden destinar las ayudas. Así, en la medida en que se limite el uso de éstas a viviendas cuyo precio no supere un determinado límite, menores posibilidades hay de que el incentivo se capitalice, sobre todo si además de esta limitación existe una normativa urbanística y de calidad en la edificación que impida que promotores y constructores traten de «apropiarse» de dicho incentivo disminuyendo la calidad de las viviendas y, con ello, su coste ${ }^{17}$.

c) El número de familias potencialmente beneficiarias de las ayudas (AlBI, GonZÁlez-PÁRAmo y Zubiri, 2000, p. 361). Cuanto mayor sea éste, se estará favoreciendo un mayor crecimiento de la demanda, con lo que los precios tenderán a subir más y, con ello, la capitalización del incentivo será mayor.

Respecto al tipo de ayudas que pueden verse afectadas con más intensidad por este fenómeno, los dos últimos factores señalados nos permiten afirmar que ésta tenderá a ser mayor cuanto más generalizadas o menos selectivas sean. En este sentido, actualmente lo serían las indirectas o fiscales.

\section{Consideraciones finales}

La visión panorámica del mercado de la vivienda que, desde este artículo, se ha intentado ofrecer nos permite realizar, a modo de resumen, las siguientes consideraciones al respecto:

En primer lugar, el interés que suscita el estudio del mercado de la vivienda encuentra su motivación tanto en las propias características del bien que en él es objeto de intercambio - que lo convierten en esencial para el desarrollo de una vida dignacomo en el papel que desempeña su construcción en el seno de la economía de un país - clave por el empleo que genera y por ser una actividad inductora de otras muchas-. Por lo tanto, existen razones sociales y macroeconómicas que justifican sobradamente su análisis.

Por otra parte, el funcionamiento del mercado de la vivienda se caracteriza por la existencia de «fallos» en el mismo y por las fuertes oscilaciones de carácter cíclico que presenta, íntimamente ligadas a la evolución de la coyuntura económica general. Estos aspectos problemáticos, unidos a la ya mencionada importancia del bien, han llevado a las Administraciones públicas de la mayor parte de los países a intervenir activamente en el mismo, convirtiéndose esta circunstancia en uno de los rasgos más destacables que presenta dicho mercado en la actualidad.

Las características de las políticas de vivienda que habitualmente se desarrollan dependen, lógicamente, de los objetivos que se persigan y del grado de prioridad que se otorgue a los mismos. Éstos suelen ser, básicamente, de tres tipos: macroeconómicos, sociales y urbanísticos. En este sentido, hay que destacar que el análisis de los instrumentos de política de vivien$\mathrm{da}$ - tanto en lo que se refiere a las normas reguladoras como, sobre todo, al sistema de ayudas establecido- es, sin duda, la mejor forma de conocer, implícitamente, dichas prioridades, que pueden no coincidir precisamente con las explicitadas por las autoridades gubernamentales. A este respecto, conviene señalar aquí que, además de los ya mencionados, es muy probable que existan también objetivos politico-electorales (ROSEN, 1987, p. 380; JAÉN y MoLINA, 1993, p. 109): dado el gran número de agentes que pueden verse beneficiados por esta política (demandantes, promotores-constructores de las viviendas, funcionarios gestores del sistema de ayudas) puede ser un buen instrumento para mejorar los resultados en este sentido.

Finalmente, no debemos olvidarnos que la política de vivienda está sometida a un cierto grado de controversia tanto en lo referente a los objetivos como a los instrumentos, al igual que ocurre con otras políticas públicas. Este hecho nos debe de servir para recordar la evidente necesidad de realizar estudios sobre el grado de eficacia del sector público en la resolución de los fallos y problemas que se observan en los distintos mercados y, en nuestro caso, en el de la vivienda. 
* Departamento de Economía Aplicada. Universidad de Oviedo.

1 Para un análisis más detallado de algunas de estas características, así como de otras no citadas aquí, véase López GarCía (1991, pp. 6-8).

${ }^{2}$ Precisamente, algunos de los más recientes estudios económicos sobre vivienda tratan de estimar el precio implícito de las distintas características de dicha mercancía, y cuya suma es el precio de mercado de este bien. A este respecto, hay que destacar muy especialmente el modelo de precios hedónicos desarrollado por Rosen (1974, pp. 34-55), el cual constituye una referencia fundamental en estos estudios, parte importante de los cuales aparece recogida en JAÉN y MOLINA (1995, pp. 73-105).

${ }^{3}$ En Taltavull (1992, p. 195) podemos encontrar una aproximación, referida a nuestro país, de dichos efectos de arrastre.

4 Téngase en cuenta, no obstante, que, descontado el efecto de la inflación, el estancamiento en términos nominales supone una reducción en términos reales.

s El razonamiento que hemos realizado en este apartado nos lleva a la conclusión de que el sector inmobiliario tiene carácter pro-cíclico —es decir, evoluciona en la misma dirección que los ciclos de la economía en su conjunto- debido a la importancia determinante que la variable renta tiene sobre la demanda de viviendas tanto de forma directa como indirecta, según hemos señalado anteriormente. Esto es lo que se observa desde hace algunas décadas en España y la mayor parte de los países occidentales (TALTAVULL, 1996, p. 98). No obstante, hay otras variables, fundamentalmente financieras, cuya evolución puede hacer que el sector inmobiliario tenga carácter contra-cíclico si sus efectos más que compensan a los de la variable renta, como lo pueden los tipos de interés o la disponibilidad de crédito (Fallis, 1985, p. 98).

${ }^{6}$ Pese a todo, estas imperfecciones no son incluidas en muchos de los modelos econométricos utilizados para analizar empíricamente este mercado, siendo tratado en esos casos como si fuese competitivo (ARNOTT, 1987).
${ }^{7}$ Desde este punto de vista, los cambios en la coyuntura económica se traducirian en una alteración de dicha tasa, que sería superior a la «natural» en épocas de recesión e inferior en épocas de expansión.

${ }^{3}$ Una aproximación más precisa al concepto de equidad, cuyo mejor sinónimo no es «igualdad», sino «justicia social», puede verse en LE GRAND (1993).

${ }^{y}$ Cosa que no se producirá si se aprovechan los procesos de renovación para expulsar de esas zonas a las capas más desfavorecidas de la sociedad sin ofrecerles una alternativa al cambio.

${ }^{10}$ En definitiva, más inelástica será dicha oferta.

${ }^{1}$ Por tanto, más que beneficiar a los inquilinos frente a los arrendadores, en realidad benefician a aquellas familias que ya han accedido a una vivienda de este tipo al establecerse el control frente a generaciones posteriores de familias potenciales usuarias de esta modalidad de tenencia (Mills y Hamilton, 1994, p. 271).

$12 \mathrm{O}$ en el alquiler, en caso de arrendamiento.

${ }^{13}$ Téngase en cuenta que no es fácil utilizar las ayudas indirectas o fiscales para desarrollar una política de este tipo, ya que muchas de estas familias no tributan por el impuesto sobre la renta personal.

${ }^{14}$ Aunque los autores citados utilizan este criterio sólo para clasificar las ayudas dirigidas a las familias con bajos ingresos, puede extenderse sin dificultad a todas las concedidas.

15 Se trata, por tanto, de ayudas poco equitativas horizontalmente (LóPEZ, 1991, pp. 42-44).

${ }^{16}$ En definitiva: transforma demanda potencial en demanda efectiva.

${ }^{17}$ No obstante, a veces puede ser difícil impedir que este fenómeno se manifieste si los vendedores tratan de exigir «sobreprecios clandestinos» -ilegales, por lo tanto- a los compradores, es decir, un pago adicional que no aparece incluido en el precio que figura en el contrato de compraventa o en el de alquiler.

\section{Bibliografía}

Albi, E.; González-Páramo, J. M., y Zubiri, I. (2000), Economía Pública I, Barcelona: Ariel.

ArNotr, K. (1987), «Economic Theory and Housing», en E. S. MiLLs, Handbook of Regional and Urban Economics, Amsterdam: North.Holland: 959.988.

Fallis, G. (1985), Housing Economics, Canada: Batterworth \& Co.

González-Páramo, J. M., y Onrubia, J. (1992), «El gasto público en vivienda en España», Hacienda Pública Española, Nos. 120-121: 189-217.

JaÉn García, M., y Molina Morales, A. (1993), «La intervención gubernamental en el mercado de la vivienda», Hacienda Pública Española, No. 127: 103-114.

- (1995): Modelos econométricos de tenencia y demanda de vivienda, Universidad de Almería, Servicio de Publicaciones.

LE Grand, J. (1993), «Economía, igualdad y justicia social», en VV.AA., I Simposio sobre igualdad y distribución de la renta y la riqueza, Madrid: Fundación Argentaria.

Leal Maldonado, J. (coord.) (1990), Informe para una nueva política de vivienda, Madrid: Servicio de Publicaciones del MOPT.

Levenfeld, G. (coord.) (1989), La vivienda y el suelo, Madrid: Comisión del programa 2000 del PSOE.

Lopez Garcia, M. A. (1991), Algunos aspectos de la economía y la política de vivienda, Departament d'Economia Aplicada, Universitat de Barcelona.
Martínez Serrano, J. A. (1982), Economía española 1960-1980: crecimiento y cambio estructural, Madrid: H. Blume.

McDonald, J. F. (1997), Fundamentals of Urban Economics, Prentice-Hall, New Yersey.

Mills, E. S., y Hamilton, B. W. (1994), Urban Economics, New York: Harper Collins College Publishers.

NichOls, A. L., y ZeCKAUSER, R. J. (1982), «Targeting Transfers through Restrictions on Recipients», The American Economic Review, vol. 72, No. 2: 372-377.

Rafols I Esteve, J. (1989), «Factores explicativos de la expansión del sector inmobiliario», Revista de Economia, No. 1: 90-92.

RodRíGuez LóPEZ, J. (1982), «Una aproximación empírica al sector de la vivienda», en R. Martín Mateo (coord.), El sector vivienda. Metodología analítica, Madrid: Instituto de Estudios de Administración Local: 183-201.

- (1990), «Innovaciones reales y potenciales en el mercado hipotecario de los noventa», Papeles de Economia Española, No. 44: 184-192.

Rosen, H. (1974), «Hedonic Prices and Implicit Markets: Product Differentiation in Pure Competition», Journal of Political Economy, No. 82: 34-55.

-- (1987), «Housing Subsidies», en A. J. Auerbach y M. Feldstein (eds.), Handbook of Public Economics, Amsterdam: North-Holland: 375-420. 
Santtago de Pablo, F. J. (1982), «La reactivación de la construcción en un mundo inflacionario», Papeles de Economía Española, No. 10: 164-175.

Smith, L.; Rosen, K., y Fallis, G. (1988), «Recent Developments in Economic Models of Housing Markets», Journal of Economic Literature, vol. XXVI: 29.64

TALTAVull de la PAZ, P. (1992), «El sector de la construcción, motor de la expansión económica reciente: presente y futuro», en Papeles de Economía Española:
Economía de las Comunidades Autónomas, Fundación de las Cajas de Ahorro Confederadas (Funcas), No. 11 (El arco mediterráneo): 193-211.

- (1996), «El sector de la construcción y la reactivación económica», Economistas, No. 74: 96-101.

WhITE, M. J., y WhITE, L. J. (1977), «The Tax Subsidy to Owner-Occupied Housing: Who Benefits?», Journal of Public Economics, 3: 111-126. 